\title{
A Power Generation Center with Back-to-back Converter Considering Post-fault Operation for MEA Application
}

\author{
Xiaoyu Lang \\ The University of Nottingham \\ Power Electronics, Machines and \\ Control Group (PEMC) \\ Nottingham, UK \\ Xiaoyu.Lang@nottingham.ac.uk \\ Patrick Wheeler \\ The University of Nottingham \\ Power Electronics, Machines and \\ Control Group (PEMC) \\ Nottingham, UK \\ pat.wheeler@nottingham.ac.uk
}

\author{
Tao Yang \\ The University of Nottingham \\ Power Electronics, Machines and \\ Control Group (PEMC) \\ Nottingham, UK \\ Tao.Yang@nottingham.ac.uk \\ Serhiy Bozhko \\ The University of Nottingham \\ Power Electronics, Machines and \\ Control Group (PEMC) \\ Nottingham, UK \\ serhiy.bozhko@nottingham.ac.uk
}

\author{
Hossein Balaghi Enalou \\ The University of Nottingham \\ Power Electronics, Machines and \\ Control Group (PEMC) \\ Nottingham, UK \\ eexhb3@exmail.nottingham.ac.uk
}

\begin{abstract}
This paper proposes a new power generation centre (PGC) for the engine of more electric aircraft (MEA) application. In this PGC, a starter/generator is connected to the high-pressure (HP) spool to start the engine in the start process and generate electric power in the other working modes, and a generator is attached to the low-pressure (LP) spool. Their outputs supply a common DC bus via active rectifiers. A backto-back (B2B) converter is deployed to connect the $\mathrm{AC}$ sides of the two generators. There are several benefits by introducing such a B2B converter into the system, and one of the major attractive points is that the $B 2 B$ converter provides an additional power flow path to the generators under HP or LP converter fault scenarios hence improving the post fault operation ability. The characteristic of post fault operation ability of the proposed PGC will be articulated in this paper. Besides, modelling, analysis and controller design of the system will be illustrated in detail. The effectiveness of proposed power generation center and its controllers is verified in Simulations.
\end{abstract}

Keywords- Modelling, more electric aircraft, power generation control, back to back converter, post fault operation, power generation centre.

\section{INTRODUCTION}

The more electric aircraft (MEA) concept is one of the major trends towards modern aerospace industry aiming for gas emission reduction, decreased fuel consumption, low maintenance cost and etc. [1]. Existing pneumatic, hydraulic and mechanical actuators should be replaced by their electrical counterparts on-board MEA. As a consequence, the on-board installed electrical power increases significantly and this results in high electrical power demand. However, traditionally, only high pressure (HP) shaft is used for the exploitation of electric power by linking a generator on it through an accessory gearbox as the speed of HP shaft is relatively high and constant. But it has been pointed out that extracting power only from HP spool could have negative influence on the performance of engine system by analysing the compressor map [6]-[7]. If too much power is taken from the HP shaft, the surge phenomenon of compressor will happen. Although the risk of surge can be lowered by bypassing excess air to the fan discharge duct, whereas this action will lead to the waste of fuel and thrust.

An alternative way is to use the low pressure (LP) shaft as an additional power source for the electric power generation, i.e. adding another generator to the LP shaft. This gives a twogenerator-single-bus structure. Different types of generators have been used as LP shaft generators so far, like wound-field synchronous machine (WFSM), induction machine (IM) and etc. [8]-[9]. However, compared with them, the permanent magnet generators (PMGs) are much more desirable in terms of power density, volume and weight. Hence the structure that two PMGs are respectively connected to the LP and HP shaft are proposed in [2], [4] as shown in Fig.1. This power generation centre is able to effectively extract power from both shafts of the engine and the two generators supply power to a common DC bus, which is more completive than AC bus since no reactive power compensation equipment is needed.

However, the PGC shown in Fig.1 has some drawbacks which undermine the feasibility in practical application. The first issue is related to the flux weakening (FW) operation of the generator coupled to the HP shaft. At full thrust settings, the rotary speed of HP spool can reach $20000 \mathrm{rpm}$ [3]. Therefore, FW control should be applied to HP generator to decrease the flux density in the air gap, hence diminishing the back EMF. Another issue is the fault tolerance ability. For the existing PGC in Fig.1, if contingency occurs to rectifiers, the system performance will be highly deteriorated and may lead to instability. For example, if open-switch fault occurs to HP rectifier, which is caused by thermal cycling extremely high collector current and gate driver fault [10], the current will distort, and can generate secondary problems such as other parts reaching breakdown [11]. In that case, stopping the operation of HP rectifier is an effective solution. However, HP generator cannot be stopped and appropriate measures should be taken to guarantee its normal operation.

To resolve the aforementioned two issues of the existing PGC in Fig.1, a new PGC containing a back-to-back (B2B) converter is proposed as shown in Fig.2. The B2B converter is applied to connect the AC sides of two generators. The idea was initially proposed by authors recently in [5]. But the potential of this PGC allowing the post fault operation of

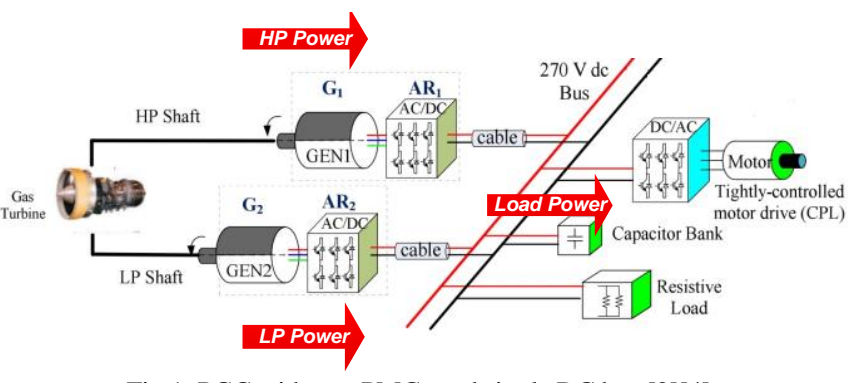

Fig.1. PGC with two PMGs and single DC bus [2][4]. 


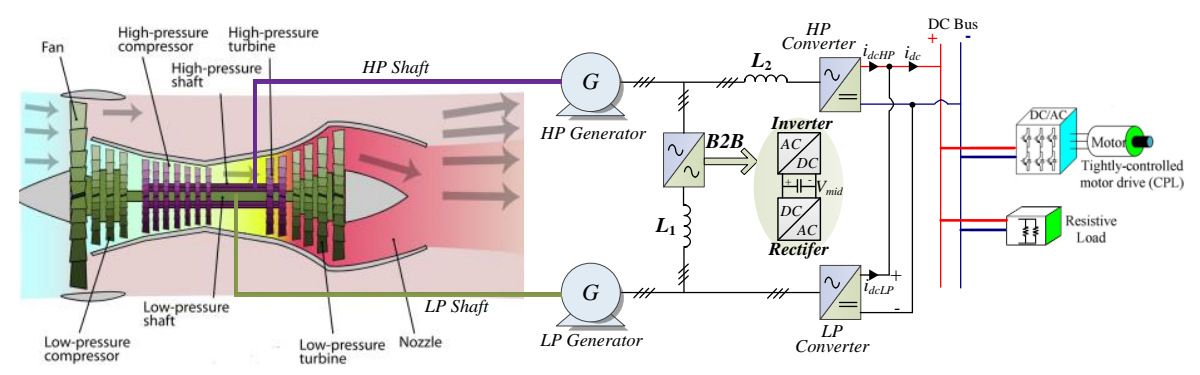

Fig.2. Proposed PGC with a back-to-back converter.

the whole system was not studied in that paper. Hence in this paper, the post fault operation ability of the proposed PGC is the main point and will be thoroughly studied considering the case that HP Converter shutdown.

This paper is organized as follows. Section II will describe the structure of the proposed PGC in detail and analyse the requirement in the post fault operation condition. Section III illustrates the modelling and controller design of the whole system, including voltage controller design, power sharing method, inductor selection and etc. In Section IV, the performances of proposed PGC will be verified. Section V makes a conclusion of the whole paper.

\section{Describtion OF the Proposed POWER GENERATION CENTRE}

As can be seen in Fig.2, a B2B converter is deployed to connect the three phases of the two generators. Since the LP generator generally outputs more power than HP generator, the $\mathrm{B} 2 \mathrm{~B}$ converter mainly serves to transfer power from the $\mathrm{LP}$ side to HP side, therefore the AC/DC and the DC/AC converters in the $\mathrm{B} 2 \mathrm{~B}$ converter are entitled as $\mathrm{B} 2 \mathrm{~B}_{\text {Rectifier }}$ and $\mathrm{B} 2 \mathrm{~B}_{\text {Inverter }}$, respectively. The rated voltage of the main DC bus in the aerospace applications is $270 \mathrm{~V}$, as described in the standard MIL-STD-704F. An interesting point of the proposed PGC is that the intermediate voltage $V_{\text {mid }}$ in the B2B converter can be set higher than $270 \mathrm{~V}$ as it doesn't need to supply onboard loads directly. Hence a relatively high $V_{\text {mid }}$ is beneficial to reduce the power loss in the B2B converter path. Moreover, two groups of inductors, denoted as $L_{1}$ and $L_{2}$ respectively, are deployed to filter the high frequency PWM harmonics and separate voltage sources, i.e., LP Converter and B2 $B_{\text {Rectifier, }}$ $\mathrm{HP}$ Converter and $\mathrm{B} 2 \mathrm{~B}_{\text {Inverter. Compared with the existing }}$ PGC in Fig.1, the proposed PGC is advantageous in the following two aspects:

1) With a high DC voltage $V_{\text {mid }}$, the HP generator is able to operate without $\mathrm{FW}$ as a high $\mathrm{AC}$ voltage can be generated

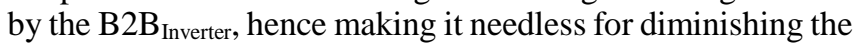
air gap flux density in the HP generator. This will be beneficial to reduce the power losses of HP generator, especially the copper loss.

2) When the LP Converter or HP Converter shutdown due to faults, like open circuit faults, unlike the existing PGC in Fig. 1 that the corresponding generators have to be cut off from the system, in the proposed PGC the B2B converter can still provide an additional power flow path for the LP or HP generators, therefore the two generators can provide continuous power to the on-board loads. Thus the post fault operation ability is greatly improved.

The initial idea of this PGC has been presented in [5], however, the point of post fault operation was not mentioned in it. Hence the main point of this paper is about post fault operation using the proposed PGC. Controller design and validations in the fault scenario will be demonstrated below.

\section{MODELLING AND CONTROLLERS DESIGN IN THE HP CONVERTER SHUTDOWN SCENARIO}

Here the scenario that HP Converter shutdown is considered. The controller design methodology when LP Converter shutdown is more or less the same as this case hence won't be illustrated due to limited space. The power flow diagram when HP Converter shutdown with the proposed PGC is shown in Fig.3. In Fig.3, there are three converters left in the system, B2 $B_{\text {Inverter }}, \mathrm{B} 2 \mathrm{~B}_{\text {Rectifier, }}$ and LP Converter. The basic structures of their controllers are discussed below:

1) For the controller of $\mathrm{B} 2 \mathrm{~B}_{\text {Inverter, there should be two }}$ cascaded loops, the outer loop is responsible for stabilizing the voltage $V_{\text {mid }}$ inside the B2B converter, and inner current loop is to control the HP generator $d q$ axes currents.

2) For the controller of $B 2 B_{\text {Rectifier, there should be two }}$ major function. The first one is to make sure the phase current of LP generator and phase current of $L_{1}$ in phase. The second is to manipulate the magnitude ratio of the phase currents of LP generator and phase current of $L_{1}$ to transfer a given amount of power via the $\mathrm{B} 2 \mathrm{~B}$ converter.

3) For the controller of LP Converter, an outer voltage loop is required for controlling the main DC bus voltage. And the inner loop is supposed to be implemented to control the $d q$ axes currents of LP generator.

The details of design process of each individual controllers are given in the following.

\section{A. Control Strategy of B2B $B_{\text {Inverter }}$}

According to the standard MIL-STD-704F [12], the nominal voltage of the main bus is $270 \mathrm{~V}$, and the acceptable steady state range is between $250 \mathrm{~V}$ and $280 \mathrm{~V}$. However, the DC voltage inside the $\mathrm{B} 2 \mathrm{~B}$ converter which is denoted as $V_{\text {mid }}$ could be adjustable since it is not used to supply the loads directly. A high $V_{\text {mid }}$ will be beneficial to reduce the current under the same power requirement, hence decreasing power loss. Therefore, in this paper, $V_{\text {mid }}$ is set as $400 \mathrm{~V}$.

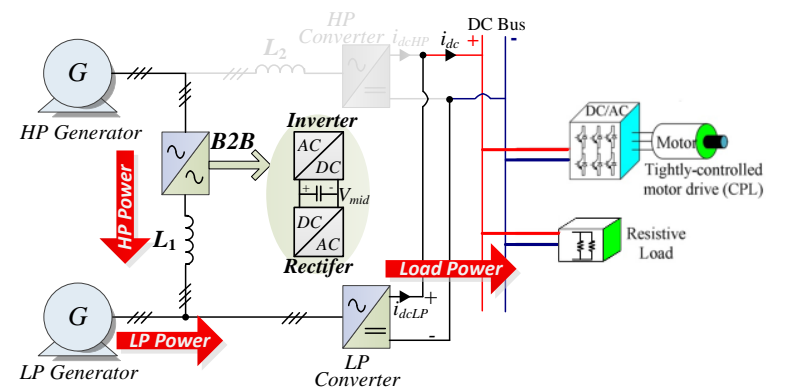

Fig.3. The power flow diagram when HP Converter shutdown with the proposed PGC. 


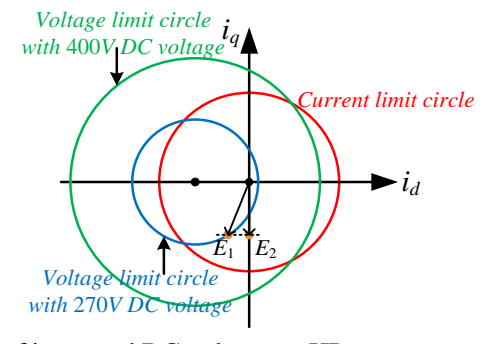

Fig.4. Effect of increased DC voltage on HP generator operating state.

For a given generator speed, the voltage limit circle radius is proportional to the DC voltage. The advantage of selecting $V_{\text {mid }}$ as $400 \mathrm{~V}$ is that HP generator could operate in a high speed without flux weakening, i.e. $i_{d}$ could be controlled as 0 . As shown in Fig.4, the equilibrium operation point moves to $E_{2}$ form $E_{1}$ with an increased DC voltage. Hence, the stator current of the HP generator decreases and the resistive losses, or copper loss within the HP generator and the HP Converter conduction loss will also reduce.

Besides the contribution of power loss reduction, the HP generator could generate relatively larger torque with the same maximum current magnitude limitation since more $i_{q}$ can be applied for the account of reduced $i_{d}$. However, the negative impact is that the switching device will withstand a higher blocking voltage.

The control block diagram of the $\mathrm{B} 2 \mathrm{~B}_{\text {Inverter }}$ is shown in Fig.5. The controller structure is very straightforward, with two cascaded loop where the outer one is voltage loop for $V_{\text {mid }}$ control and the inner one is current loop for the $d q$ axes current regulation of HP generator. Since the FW operation of HP generation is eliminated due to a high $V_{\text {mid }}$, the $d$ axis current reference is set as 0 for maximizing efficiency. The details about PI gains selection can refer to the published papers of our group [4]-[5], which are supposed to be adaptive according to the operation points, e.g. DC voltage and load power.

\section{B. Control Strategy of LP Converter}

As regards to the LP generator, its operating state should keep unchanged even after the reconfiguration of system, i.e., its speed, torque, $d q$ axes should remain unchanged. Therefore, it is obvious that the terminal voltages, or line-toline voltages of LP generator should be kept as the same as before. To fulfil this goal, the controller of LP Converter should keep unchanged as well. The aim of outer voltage loop is to keep the main DC bus as $270 \mathrm{~V}$, and the aim of inner current loop is to generate appropriate $d q$ axes currents for the operation of LP generator. The diagram of LP Converter controller should be the same as that of $\mathrm{B} 2 \mathrm{~B}_{\text {Inverter shown in }}$ Fig.5. However, two points should be modified:

1) For the controller of LP Converter, the voltage reference should be changed from $V_{\text {mid }}$ to $V_{d c}$.

2) For the controller of LP Converter, the $d q$ axes current feedbacks should not be the currents of LP generator but the $d q$ axes currents of the LP side main branches.

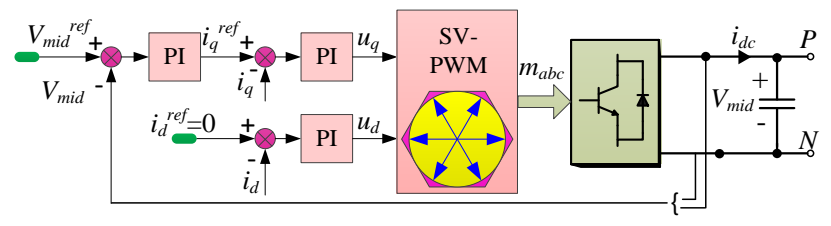

Fig.5. Control block diagram of the $\mathrm{B}_{2} \mathrm{~B}_{\text {Inverter }}$.

\section{Control Strategy of $B 2 B_{\text {Rectifier }}$}

The inductor $L_{1}$ shown in Fig.2 is important due to its following functions: 1) separate $u_{i n v}$ and $u_{\text {rec }}$, and make $u_{i n v}$ adjustable in terms of specific conditions; 2) filter the high frequency PWM harmonics in the currents. Therefore, the selection criterion of inductor value should be discussed first.

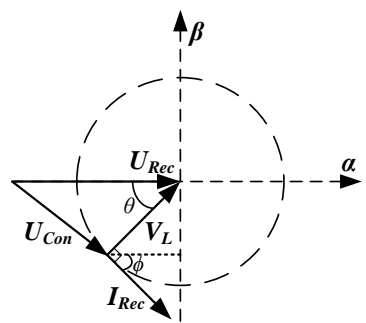

Fig.6. Relationship of voltage vectors.

The relationship of voltage vectors is shown in Fig.6, where $\boldsymbol{U}_{R e c}$ represents voltage generated by $\mathrm{B} 2 \mathrm{~B}_{\text {Rectifier, }}, \boldsymbol{V}_{L}$ is voltage across inductor, and $\boldsymbol{U}_{C o n}$ is voltage generated by LP Converter, respectively. According to cosine theorem, the following equation can be obtained:

$$
\begin{aligned}
\left|U_{C o n}\right|^{2} & =\left|U_{R e c}\right|^{2}+\left|V_{L}\right|^{2}-2\left|U_{R e c}\right|\left|V_{L}\right| \cos \theta \\
& =\left|U_{R e c}\right|^{2}+\left|V_{L}\right|^{2}-2\left|U_{R e c}\right|\left|V_{L}\right| \sin \phi
\end{aligned}
$$

Since $V_{L}=j \omega L I_{R e c}$, therefore (1) can be rewritten as

$$
\left|U_{C o n}\right|^{2}=\left|U_{R e c}\right|^{2}+\omega^{2} L^{2}\left|I_{R e c}\right|^{2}-2 \omega L\left|U_{R e c}\right|\left|I_{R e c}\right| \sin \phi
$$

where $L$ represents the value of inductor per phase.

Choose $L$ as the variable, then $L$ can be obtained as follows

$$
\begin{aligned}
& L=\frac{\left|U_{R e c}\right| \sin \phi+\sqrt{\left|U_{R e c}\right|^{2} \sin ^{2} \phi+\left|U_{C o n}\right|^{2}-\left|U_{R e c}\right|^{2}}}{\omega\left|I_{R e c}\right|} \\
& =\frac{U_{R e c_{-} m} \sin \phi+\sqrt{U_{R e c_{-} m}{ }^{2} \sin ^{2} \phi+U_{C o n_{-} m}{ }^{2}-U_{R e c_{-} m}{ }^{2}}}{\omega I_{R e c_{-} m}}
\end{aligned}
$$

where $U_{R e c \_m}, U_{\text {Con_m }}, I_{R e c \_m}$, represent the maximum value of $\boldsymbol{U}_{R e c}, \boldsymbol{U}_{C o n}$ and $\boldsymbol{I}_{R e c}$, respectively.

The typical speed of LP generator is $4000 \mathrm{r} / \mathrm{min}$, according to the machine's parameters shown in Table I, the electrical speed $\omega_{e}=1256 \mathrm{rad} / \mathrm{s}$, and the back EMF $E_{0}=\omega_{e} \psi_{f}=45.8 \mathrm{~V}$. Since the stator inductance is rather small, the maximum value of $\boldsymbol{U}_{\text {Con }}$ is similar to $E_{0}, U_{\text {Con } \_} \approx E_{0}=45.8 \mathrm{~V}$. The maximum current permitted of each rectifier in this paper is $400 \mathrm{~A}$, and generally LP accounts for more power than HP generator, therefore assume $I_{\text {Rec } m}$ is $1 / 4$ maximum, which is $100 \mathrm{~A}$. Besides, assume $U_{R e c \_m}$ is $100 \mathrm{~V}$ and $\phi=70$, in this case, $L$ can be obtained: $L<1.2 \mathrm{mH}$.

\section{TABLE I. MAIN PARAMETERS OF LP SG}

\begin{tabular}{cc}
\hline \hline parameter & rated value \\
\hline Motor Power & $45 \mathrm{~kW}$ \\
Poles & 3 \\
Stator Resistance & $0.015 \mathrm{Ohm}$ \\
Stator Inductance & $0.1 \mathrm{mH}$ \\
Flux Linkage of Magnet & $0.0365 \mathrm{~Wb}$ \\
\hline \hline
\end{tabular}




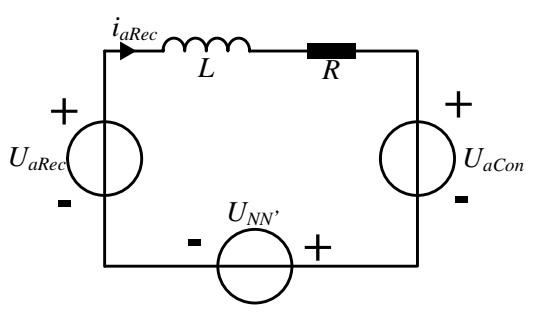

Fig.7. The equivalent circuit model of $a$ phase.

It should be noted that the threshold value of $L$ changes in terms of operating conditions, like load, generator speed, power sharing ratio and etc. Therefore, in the simulation of this report, $L$ is chosen as $0.8 \mathrm{mH}$ which is an appropriate value.

Next is to controller model of $\mathrm{B} 2 \mathrm{~B}_{\text {Rectifier. The equivalent }}$ circuit model of $a$ phase is demonstrated as Fig.7, where $N$ is reference point of LP Converter, $N^{\prime}$ is the reference point of $\mathrm{B} 2 \mathrm{~B}_{\text {Rectifier. }}$

Since LP generator and $\mathrm{B} 2 \mathrm{~B}_{\text {Rectifier }}$ system share common junctions, an effective way to fulfill power sharing between LP generator and HP generator is to control $i_{L}$ and $i_{R e c}$ with same phase displacement. The ratio of magnitude depends on the particular power sharing ratio.

According to Fig.7, the following equations can be obtained

$$
\left\{\begin{array}{l}
P=\sum_{k=a, b, c} u_{k R e c} i_{k R e c} \\
u_{k R e c}=R i_{k R e c}+L \frac{d i_{k R e c}}{d t}+u_{k C o n}+u_{N N^{\prime}}, k=a, b, c \\
\sum_{k=a, b, c} i_{k R e c}=\sum_{k=a, b, c} u_{k C o n}=0
\end{array}\right.
$$

Then the voltage difference between LP Converter reference point $N$ and $\mathrm{B} 2 \mathrm{~B}_{\text {Rectifier }}$ reference point $N^{\prime}$ is

$$
u_{N N^{\prime}}=\frac{1}{3} \sum_{k=a, b, c} u_{k R e c}
$$

Apply Clark transformation ( $3 / 2$ transformation) to (4) and adopt (5), the mathematical model in $\alpha \beta$ coordinates can be derived as follows

$$
\left\{\begin{array}{l}
P=\frac{3}{2}\left(u_{\alpha R e c} i_{\alpha R e c}+u_{\beta R e c} i_{\beta R e c}\right) \\
u_{\alpha R e c}=R i_{\alpha R e c}+L \frac{d i_{\alpha R e c}}{d t}+u_{\alpha C o n} \\
u_{\beta R e c}=R i_{\beta R e c}+L \frac{d i_{\beta R e c}}{d t}+u_{\beta C o n}
\end{array}\right.
$$

Then apply rotating factor $e^{j \theta}(\theta=\omega t)$ to (6), the mathematical model in $d q$ coordinates is derived

$$
\left\{\begin{array}{l}
P=\frac{3}{2}\left(u_{d R e c} i_{d R e c}+u_{q R e c} i_{q R e c}\right) \\
u_{d R e c}=R i_{d R e c}+L \frac{d i_{d R e c}}{d t}+u_{d C o n}-\omega L i_{q R e c} \\
u_{q R e c}=R i_{q R e c}+L \frac{d i_{q R e c}}{d t}+u_{q C o n}+\omega L i_{d R e c}
\end{array}\right.
$$

Then the $d q$ axes current controller of $\mathrm{B} 2 \mathrm{~B}_{\text {Rectifier with }}$ decoupling terms can be obtained, and demonstrated in Fig.8.

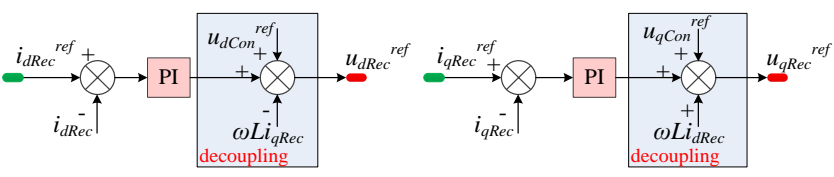

Fig.8. $d q$ axes current controller of $\mathrm{B} 2 \mathrm{~B}_{\text {Rectifier }}$ with decoupling terms.

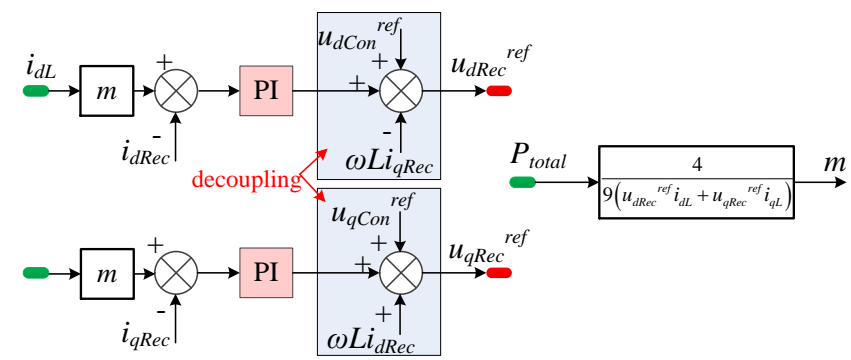

Fig.9. Overall control diagram of $\mathrm{B} 2 \mathrm{~B}_{\text {Rectifier. }}$.

Next question is how to acquire the $d q$ axes current reference in order to realize power sharing between HP and LP generators. This problem is addressed based on controlling $i_{L}$ and $i_{R e c}$ in phase. In this paper, the power sharing ratio between LP and HP is $2: 1$. Then the following relation of power can be obtained:

$$
\frac{3}{2}\left(u_{d R e c} i_{d R e c}+u_{q R e c} i_{q R e c}\right)=\frac{2}{3} P_{\text {total }}
$$

where $P_{\text {total }}$ is the total power consumption. $P_{\text {total }}=V_{d c} I_{o}$, where $V_{d c}$ is the main DC bus voltage which is kept as $270 \mathrm{~V}$, and $I_{o}$ is the DC bus current which can be measured.

Assume the magnitude relation between $i_{L}$ and $i_{R e c}$ is $i_{R e c}=m i_{L}$, where $m$ is a ratio, then (8) can be rewritten as

$$
\begin{aligned}
& \frac{3}{2} m\left(u_{d R e c} i_{d L}+u_{q R e c} i_{q L}\right)=\frac{2}{3} V_{d c} I_{o} \\
& \Rightarrow m=\frac{4 V_{d c} I_{o}}{9\left(u_{d R e c} i_{d L}+u_{q R e c} i_{q L}\right)}
\end{aligned}
$$

Then the control diagram of $\mathrm{B}_{2} \mathrm{~B}_{\text {Rectifier }}$ can be established as shown in Fig.9.

\section{VALIDATIONS}

In this section, simulations about the control performance when the proposed PGC in normal and post fault operation conditions are conducted by the co-simulation of Simulink and PLECS. The parameters of LP and HP generators are listed in Table I. There are three operation phases: between $0 \mathrm{~s}$ to $0.1 \mathrm{~s}$, the whole system operates in normal state, which means HP Converter works normally, and the power sharing ratio between LP and HP generator is $1: 1$. At $0.1 \mathrm{~s}$, the HP Converter is cut off, then the B2B converter is applied to the system for post fault operation. After $0.2 \mathrm{~s}$, the power sharing ratio changes to $2: 1$.

The $d q$ axes currents of HP generator are shown in Fig.10(a). It can be seen that before $0.1 \mathrm{~s}, i_{d}$ is negative to realize the flux weakening operation. While after $0.1 \mathrm{~s}, i_{d}$ is kept to 0 as the DC side voltage rises from $270 \mathrm{~V}$ to $400 \mathrm{~V}$, which will benefit to reduce the copper losses. And as HP generator outputs less power after $0.2 \mathrm{~s}$, the magnitude of $i_{q}$ become smaller.

The $d q$ axes currents of LP generator are shown in Fig. 10(b). $i_{d}$ is controlled to 0 during the whole process as the speed of LP generator is relatively slow. While after $0.2 \mathrm{~s}$, the magnitude of $i_{q}$ becomes larger since it accounts for more power. In addition, the behaviour of the main DC bus 


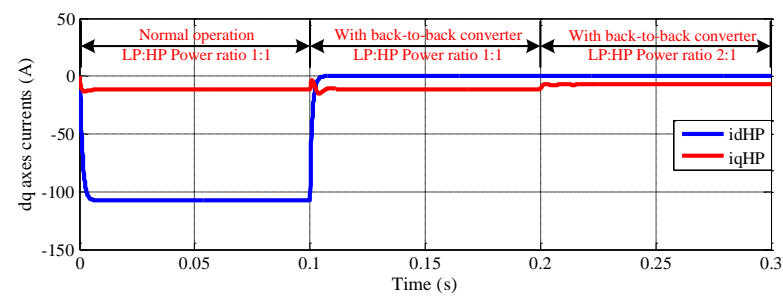

(a)

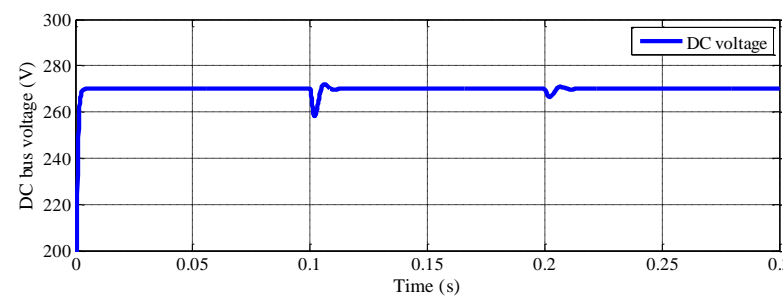

(c)

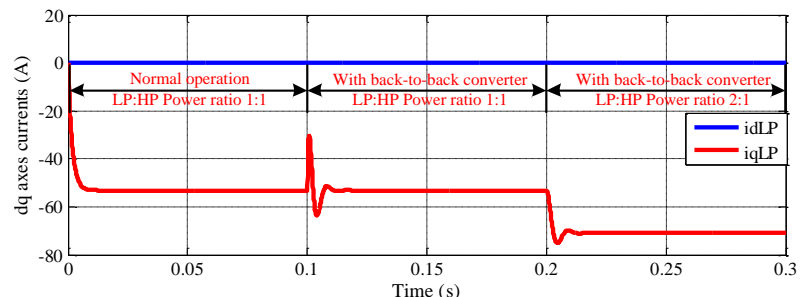

(b)

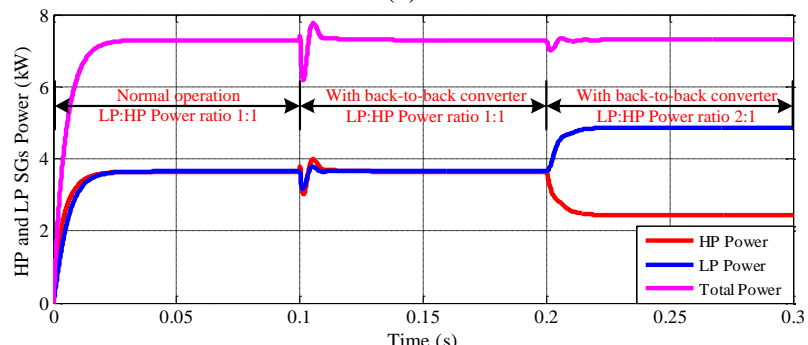

(d)

Fig.3. Performance of the proposed PGC. (a) $d q$ currents of HP generator; (b) $d q$ currents of LP generator; (c) main DC voltage; (d) Powers of LP generator, HP generator and the total output power.

voltage is kept stable with the steady value to $270 \mathrm{~V}$ as shown in Fig.10(c).

The output powers of LP generator, HP generator and total power are shown in Fig.10(d). The main DC bus keeps to $270 \mathrm{~V}$, and load is chosen as a $10 \Omega$ resistive load, then the total power is $7.3 \mathrm{~kW}$. It can be seen from Fig. 10(d) that before $0.2 \mathrm{~s}$, in the steady state LP and HP supply the load equally no matter in normal or fault condition. While after the power ratio changes to 2:1, LP power becomes twice of that of HP.

\section{CONCLUSION}

In this paper, a new power generation center (PGC) for the MEA application is proposed. Two PMGs are attached to HP and LP spools feeding a $270 \mathrm{~V}$ main DC bus. A back-to-back converter is used to transfer power between LP side and HP side. Modelling, controller design for each individual converter, and the criterion for inductor selection are given. Simulation results confirm the post fault operation ability of the proposed PGC under HP Converter shutdown. The main contributions that have been validated can be highlighted below:

1) The proposed PGC has the post-fault operation ability when any of HP or LP converters shutdown under fault scenarios since the back-to-back converter provides an additional power flow path for the generators.

2) The HP generator is allowed to operate at a high speed without flux weakening, reducing the magnitude of stator current. This will help to reduce the overall copper losses.

\section{ACKNOWLEDGMENT}

This project has received funding from the Clean Sky 2 Joint Undertaking under the European Union's Horizon 2020 research and innovation program under grant agreement No 807081. The author Xiaoyu Lang also thanks the funding from China Scholarship Council (CSC).

\section{REFERENCES}

[1] B. Sarlioglu and C. T. Morris, "More electric aircraft: Review, challenges, and opportunities for commercial transport aircraft," IEEE Trans. Transport. Electrific., vol. 1, no. 1, pp. 54-64, Jun. 2015.

[2] F. Gao, S. Bozhko, G. Asher, P. Wheeler, and C. Patel, "An improved voltage compensation approach in a droop-controlled DC power system for the more electric aircraft," IEEE Trans. Power Electron., vol. 31, no. 10, pp. 7369-7383, Oct. 2016.

[3] C. Li, T. Yang, P. Kulsangcharoen, G. L. Calzo, S. Bozhko, C. Gerada, and P. Wheeler, "A modified neutral-point balancing space vector modulation technique for three-level neutral point clamped converters in high speed drives," IEEE Trans. Ind. Electron., vol. 66, no. 2, pp. 910-921, Feb. 2019.

[4] X. Lang, T. Yang, H. B. Enalou, S. Bozhko and P. Wheeler, "An Enhanced Power Generation Centre for More Electric Aircraft Applications," 2018 IEEE International Conference on Electrical Systems for Aircraft, Railway, Ship Propulsion and Road Vehicles \& International Transportation Electrification Conference (ESARSITEC), Nottingham, 2018, pp. 1-6.

[5] X. Lang, T. Yang, H. B. Enalou, C. Li, S. Bozhko and P. Wheeler, " A Dual-Channel Enhanced Power Generation Architecture with Back-toback Converter for MEA Application," 2019 International Electrical Machine Design Conference, San Diego, CA, USA, 2019, in press.

[6] Enalou, Hossein Balaghi, et al. "Potential Improvements in Turbofan's Performance by Electric Power Transfer", No. 2018-01-1962. SAE Technical Paper, 2018.

[7] H. B. Enalou, S. Bozhko, M. Rashed, and et. al., "A Preliminary Study into Turbofan Performance with LP-HP Power Exchange," in GPPS Global Power \& Propulsion Society, Montreal, 2018.

[8] K. Muehlbauer and D. Gerling, "Two-generator-concepts for electric power generation in more electric aircraft engine," in Proc. 19 Int. Conf. Elect. Mach., 2010, pp. 1-5.

[9] Y. Jia and K. Rajashekara, "An Induction Generator-Based AC/DC Hybrid Electric Power Generation System for More Electric Aircraft," IEEE Trans. Indus. Appl., vol. 53, no. 3, pp. 2485-2494, June 2017.

[10] U. M. Choi, H. G. Jeong, K. B. Lee, and B. F. Blaabjerg, "Method for detecting an open-switch fault in a grid-connected NPC inverter system," in IEEE Trans. Power Electron., vol. 27, no. 6, pp. 27262739, Jun. 2012

[11] J. S. Lee and K. B. Lee, "Open-Switch Fault Tolerance Control for a Three-Level NPC/T-Type Rectifier in Wind Turbine Systems," in IEEE Trans. Industrial Electron., vol. 62, no. 2, pp. 1012-1021, Feb. 2015.

[12] Aircraft Electric Power Characteristics, American Military Std. MILSTD-704F, Mar. 2004. 